University of Wollongong

Research Online

Australian Institute for Innovative Materials -

Papers

Australian Institute for Innovative Materials

$1-1-2017$

Evidencing extrinsic stacking faults in twinning-induced plasticity steel

Gilberto Casillas

University of Wollongong, gilberto@uow.edu.au

Azdiar A. Gazder

University of Wollongong, azdiar@uow.edu.au

Elena V. Pereloma

University of Wollongong, elenap@uow.edu.au

Ahmed A. Saleh

University of Wollongong, asaleh@uow.edu.au

Follow this and additional works at: https://ro.uow.edu.au/aiimpapers

Part of the Engineering Commons, and the Physical Sciences and Mathematics Commons

Research Online is the open access institutional repository for the University of Wollongong. For further information contact the UOW Library: research-pubs@uow.edu.au 


\title{
Evidencing extrinsic stacking faults in twinning-induced plasticity steel
}

\author{
Abstract \\ In twinning induced plasticity (TWIP) steel, the question of what leads to the onset of twinning in grains \\ whose (100) orientations are near parallel to the tensile axis at high tensile strains has not been resolved. \\ Using aberration corrected scanning transmission microscopy we present first-hand evidence that \\ extrinsic stacking faults serve as nuclei for twins in (100) grains and also contribute to twin growth in a \\ low carbon TWIP steel uniaxially tensile loaded up to its ultimate tensile strength of $\sim 1080 \mathrm{MPa}$; which \\ corresponds to a tensile true strain of $48 \%$. The results corroborate and validate our previous Schmid \\ factor $¿$ based predictions on the feasibility of twinning nucleation via extrinsic stacking faults in (100) \\ grains at high tensile strains. \\ Disciplines \\ Engineering | Physical Sciences and Mathematics

\section{Publication Details} \\ Casillas, G., Gazder, A. A., Pereloma, E. V. \& Saleh, A. A. (2017). Evidencing extrinsic stacking faults in \\ twinning-induced plasticity steel. Materials Characterization, 123 275-281.
}




\title{
Evidencing extrinsic stacking faults in twinning-induced plasticity steel
}

\author{
Gilberto Casillas ${ }^{1}$, Azdiar A. Gazder ${ }^{1}$, Elena V. Pereloma ${ }^{1,2}$, Ahmed A. Saleh ${ }^{2 *}$ \\ ${ }^{1}$ Electron Microscopy Centre, University of Wollongong, New South Wales 2500, Australia \\ ${ }^{2}$ School of Mechanical, Materials and Mechatronic Engineering, University of Wollongong,
} New South Wales 2522, Australia

\begin{abstract}
In twinning induced plasticity (TWIP) steel, the question of what leads to the onset of twinning in grains whose $\langle 100\rangle$ orientations are near parallel to the tensile axis at high tensile strains has not been resolved. Using aberration corrected scanning transmission microscopy we present first-hand evidence that extrinsic stacking faults serve as nuclei for twins in $\langle 100\rangle$ grains and also contribute to twin growth in a low carbon TWIP steel uniaxially tensile loaded up to its ultimate tensile strength of $1080 \mathrm{MPa}$; which corresponds to a tensile true strain of $48 \%$. The results corroborate and validate our previous Schmid factor -based predictions on the feasibility of twinning nucleation via extrinsic stacking faults in $\langle 100\rangle$ grains at high tensile strains.
\end{abstract}

Keywords: scanning transmission electron microscopy (STEM); extrinsic stacking faults; intrinsic stacking faults; twinning; Schmid factor; TWIP steel

\section{*Corresponding author:}

\section{Ahmed A. Saleh}

School of Mechanical, Materials and Mechatronic Engineering

University of Wollongong, New South Wales 2522, Australia

Phone: +61242213034Ｆax: +61242213114ｅ-mail: asaleh@uow.edu.au 


\section{Introduction}

The deformation behaviour of low stacking fault energy, face-centered-cubic (fcc), high Mn twinning induced plasticity (TWIP) steel during tensile loading has been the subject of interest in recent investigations [1-4]. Over the last decade significant progress has been made in understanding the origin of twinning and their growth mechanisms ${ }^{1}$ in fcc materials. It is accepted that for coarse-grained $(>1 \mu \mathrm{m})$ fcc materials the formation of twins occurs by Shockley partial dislocations with $a / 6\langle 112\rangle$ Burgers vectors (where $a=$ lattice parameter) gliding on successive $\{111\}$ slip planes. A very detailed overview of the mechanisms of twin formation in fcc metals was undertaken by Christian and Mahajan [5]. The possible twinning mechanisms could be divided into two main groups based on their source type [5]: (i) prismatic [6-13] or, (ii) glide [14-18].

A characteristic of prismatic sources is that the partial dislocation which generates the twin is formed from dislocations with Burgers vectors that do not lie in the twin plane [5]; for example, via the pole [7] and ratchet [9] mechanisms. Although Cottrell and Bilby [7] were the first to point out the possibility of single-layer twin formation via prismatic sources, Venables [19] and Hirth [9] suggested a modification of the pole mechanism that accounted for the continuous growth of twins from a single stacking fault. Models using prismatic sources and dislocation barriers were also proposed by Cohen and Weertman [10], Mori and Fujita [11], Miura-Takamura-Narita (MTN) [12] and Niewczas and Saada [13].

On the other hand, glide sources produce twins from dislocations with Burgers vectors that lie in the twin plane. The dislocations include those produced by the interaction of glide dislocations of the same slip system [14], of primary and co-planar slip systems [16-18] or primary and cross-slip systems [15]. Mahajan and Chin [14] proposed the dissociation of two co-planar perfect dislocations $(a / 2[011]+$ $a / 2[\overline{1} 01])$ into three Shockley partial dislocations $(a / 6[\overline{1} 12])$ on two consecutive and adjacent (1111) planes resulting in an extrinsic stacking fault (ESF) that acts as a two-layered nucleus for twinning. Later

\footnotetext{
1 Throughout the text, the term growth mechanism refers to twin thickening.
} 
on Mahajan [18] suggested that twins could also form directly from fault-pairs. In the latter case, both slip bands and twins lie in the same plane.

With respect to TWIP steels subjected to tensile loading, texture and microstructure characterisation via electron back-scattering diffraction [4] has shown: (i) the characteristic development of a relatively stronger $\langle 111\rangle$ and a weaker $\langle 100\rangle$ double partial fibre texture parallel to the tensile axis (II TA). (ii) Twinning is promoted in grains whose $\langle 111\rangle$ orientations are near parallel to the tensile axis (hereafter referred to as $\langle 111\rangle$ grains) from the early deformation stages onwards. Twinning is sustained upon increasing tensile strain as more grains progressively rotate towards the $\langle 111\rangle$ fibre. (iii) Lastly, the onset of twinning in grains whose $\langle 100\rangle$ orientations are near parallel to the tensile axis (hereafter referred to as $\langle 100\rangle$ grains) occurs at relatively high tensile strains.

The nucleation and dependence of deformation twinning on grain orientation in low stacking fault energy fcc materials is typically explained using the Schmid factor [1, 4, 20-22]; which in turn, provides a geometric or crystallographic measure of the tendency of a certain orientation to deform by a particular deformation mode. Since the Schmid factor for twinning is higher than that for slip in $\langle 111\rangle$ grains, twinning activity is promoted and sustained in those grains with increased tensile loading [3]. In this regard, Schmid factor analyses of twinning [1,4] generally imply that twins nucleate via intrinsic stacking faults (ISFs); as the Schmid factor values for twinning and the leading partial of ISFs are equivalent [22]. In doing so, the onset of twinning in $\langle 100\rangle$ grains (i.e.- orientations that are nominally regarded as being unfavourable to twinning/intrinsic faulting) at high tensile strains is considered a deviation from Schmid's law and ascribed to the localised stress concentration effect induced by neighbouring grain interactions $[1,4]$.

Alternatively, Karaman et al. [21] pointed out that the Schmid factor for twinning will depend on whether an ISF or ESF acts as the twin nucleus. Since calculations considering twin nucleation from extrinsic faults were completely overlooked, we undertook Schmid factor predictions considering the leading partials of both, ISFs and ESFs [23]. In Ref. [23], we were the first to clearly demonstrate the 
feasibility of ESFs to act as twin nuclei in orientations with nominally low Schmid factors for twinning (such as $\langle 100\rangle$ grains).

Without considering grain orientation and its correlation with the macroscopic loading condition and/or direction, previous transmission electron microscopy (TEM) studies by Idrissi et al. [24, 25] identified ISFs as nuclei for deformation twinning in Fe-21Mn-1.2C deformed at room temperature and Fe-17Mn-3Al-3Si deformed at $186^{\circ} \mathrm{C}$. In Ref. [24] they reported that the dislocation reactions deviate from the pole mechanism and lead to twin nucleation [10] and that growth takes place by a secondary polar mechanism [19]. Contrarily, the same authors investigated Fe-28Mn-3.5Si-2.8Al deformed at room temperature and reported large, homogenously distributed ESFs acting as preferential sources for twinning nucleation [26] and stated that the Mahajan and Chin [14] mechanism could be operative at early strains whereas twinning through a stair-rod cross-slip mechanism [11] was operative at relatively high strains.

In summary, the question of what leads to the onset of twinning in $\langle 100\rangle$ grains at high tensile strains is not resolved and can be ascribed to two reasons: (i) that the feasibility of ESFs to serve as twin nuclei has been completely overlooked, and (ii) that none of the earlier TEM -based studies correlated their observations of both ESFs and/or ISFs acting as twin nuclei with grain orientation and the macroscopic loading condition and/or direction.

Consequently, this paper focusses on correlating the type of faulting that leads to twinning in $\langle 100\rangle$ and 〈111) grains of a low carbon TWIP steel uniaxially tensile loaded up to its ultimate tensile strength of 1080 MPa; which corresponds to a tensile true strain of 48\%. Aberration corrected scanning transmission electron microscopy (STEM) was used to identify the types of faults as well as provide hints as to the dislocation reactions that possibly led to twinning during tensile loading. Most importantly, the present results are in agreement with our previous Schmid factor -based predictions on the feasibility of twinning nucleation via ESFs and ISFs in $\langle 100\rangle$ and $\langle 111\rangle$ grains, respectively [23].

\footnotetext{
${ }^{2}$ Throughout the text, chemical compositions are in weight percent (wt. \%) unless specified otherwise.
} 
On a correlated note, it should be kept in mind that the earlier TEM-based studies provided a variety of dislocation reactions that led to twinning. In the same vein, the possible dislocation reactions shown here should be interpreted with caution as the data corresponds to a low carbon TWIP steel uniaxially tensile loaded to its ultimate tensile strength of $\sim 1080 \mathrm{MPa}$ at $48 \%$ true strain.

Moreover, the internal structure and morphology of deformation twins in TWIP steels heavily depend on the carbon content of the alloy. As demonstrated by Idrissi et al. [27], deformation twins in a high carbon Fe-20Mn-1.2C TWIP steel were finer and full of sessile dislocations compared to an Fe-28Mn3.5Si-2.8Al TWIP steel with nominally no carbon. Consequently, the results shown here must not be readily applied to high carbon TWIP steels without prior characterisation; as faults and twinning mechanisms are subject to unique alloy chemistries as well as loading conditions and strain levels.

\section{Experimental and analytical procedure}

An Fe-24Mn-3Al-2Si-1Ni-0.06C TWIP steel was slab-cast, hot-rolled at $1100{ }^{\circ} \mathrm{C}$ to $52 \%$ thickness reduction and cold-rolled to $42 \%$ thickness reduction. A flat dog-bone shaped tensile sample of $25 \mathrm{~mm}$ gauge length, $5 \mathrm{~mm}$ width and $1 \mathrm{~mm}$ thickness was wire-cut from the centre of the cold-rolled strip. The gauge length and width of the tensile sample were parallel to the rolling and transverse directions of the cold rolled strip, respectively. Following this, the tensile sample was isochronally annealed at $850{ }^{\circ} \mathrm{C}$ to its fully recrystallised state. The heat treatment included $240 \mathrm{~s}$ of heating to a stable temperature followed by $300 \mathrm{~s}$ of soaking time and immediate water quenching.

Uniaxial tensile testing was undertaken on an in-house modified Kammrath and Weiss GmbH tensile stage operating in speed control mode at $5 \mu \mathrm{m} \cdot \mathrm{s}^{-1}$. The TWIP steel was loaded up to its ultimate tensile strength of $\sim 1080 \mathrm{MPa}$ which corresponds to a tensile true strain of $48 \%$.

Extreme care was taken to ensure that each of the $\varnothing 3 \mathrm{~mm}$ discs punched out from the gauge length contained a short chord parallel to the tensile axis. The chord is necessary to facilitate the identification of 
grain orientation in the TEM. The discs were ground to $\sim 70 \mu \mathrm{m}$ thickness and twin-jet electro-polished using a solution of $90 \%$ methanol and $10 \%$ perchloric acid in a Struers Tenupol-5 operating at $30 \mathrm{~V}(\sim 150$ $\mathrm{mA}$ ) and $-30{ }^{\circ} \mathrm{C}$.

Bright field images, selected area electron diffraction (SAED) patterns, atomic resolution STEM and high-angle annular dark field (HAADF) images were acquired on a probe-corrected, cold field-emission gun JEOL JEM-ARM200CF TEM operating at $200 \mathrm{kV}$. Since the rotation between the TEM image and the SAED pattern in this particular microscope is $<1^{\circ}$, the task of identifying grain orientation is greatly facilitated. Diffraction patterns close to $\langle 110\rangle$ zone axes were first acquired; after which the direction of the macroscopic tensile axis (delineated by the chord on the electro-polished discs) was superimposed on the SAED pattern to identify grains whose $\langle 100\rangle$ and $\langle 111\rangle$ orientations were parallel to the tensile axis (Fig. 1).

The HAADF images were acquired with 50 and 180 mrad inner and outer collection angles respectively with a dwell time of $38 \mu$ s and a convergence solid angle of 25 mrad; resulting in a probe current of $\sim 35$ pA and $\mathrm{a} \sim 1 \AA$ probe diameter . 


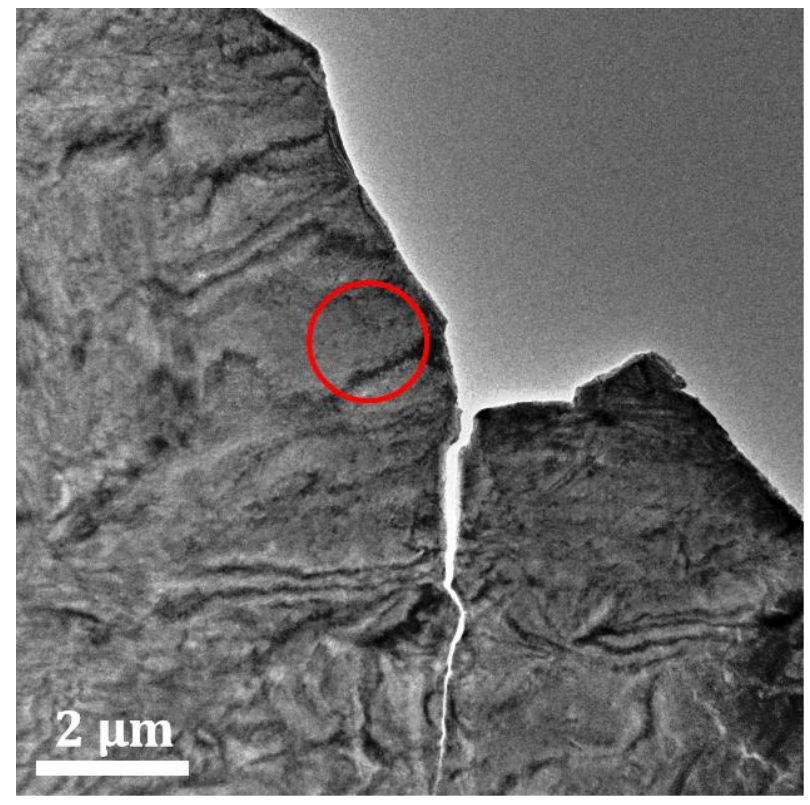

(a)

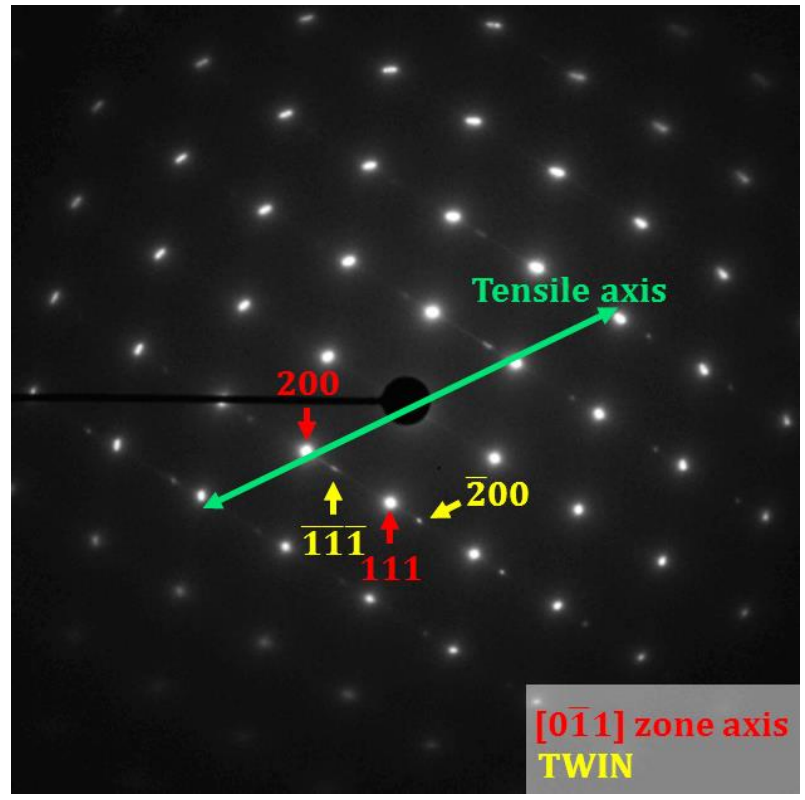

(b)

Figure 1: (a) Bright field image with a solid red circle marking a grain whose $\langle 100\rangle$ orientation is parallel to the tensile axis. The circle also denotes the area from where the SAED pattern was acquired in (b) along the $[0 \overline{1} 1]$ zone axis. The TEM foil contains a short chord marking the tensile axis; which is placed along the axis of the holder indicated by the solid green line in (b).

\section{Results}

There are inconsistencies and discrepancies in the literature related to the definition of ESFs and twin boundaries. Researchers have interchangeably referred to an ESF as either a twin [28] or a twin-nucleus of two layers [29]. A short discussion arguing the use and/or appropriateness of these terms is provided at the beginning of Section 4. However, for the purposes of the present investigation, Weertman's convention [30] is strictly applied as follows:

An ISF is defined when a fault is generated by the removal of a plane of atoms resulting in an $\mathrm{ABC} A \mathrm{CABC}$ sequence. Alternatively, when a plane of atoms is inserted into the sequence, it results in an ESF with an $A B C A|C| B C A$ sequence. These sequences can also be observed by the glide of Shockley partial dislocations on adjacent $\{111\}$ planes, however, only when two partial dislocations bound a double fault it is considered an ESF. 
A twin boundary is a plane on which one side of the crystal can be mirrored on to the other and results in an ABCABACBA sequence; with the plane $\mathbf{B}$ denoting the twin plane. Confusion arises when looking at the ESF sequence $\mathrm{ABCA}|\mathrm{C}| \mathrm{BCAB}$; where $\mathbf{A}$ and $\boldsymbol{B}$ can be thought of as twin boundaries; which in turn leads to ESFs being referred to as "micro-twins" or "twin nuclei". However, in this paper a twin boundary is delineated by at least three $\{111\}$ planes on either side of that boundary. It follows that as a minimum, one unit cell is present on either side of the twin; such that the sequence $A B C A|C B| A B C A$ is the minimum number of stacking faults inside the fcc matrix in order for both $\mathbf{A}$ and $\boldsymbol{A}$ to be considered twin boundaries.

\subsection{Twin nucleation by extrinsic stacking faults in $\langle 100\rangle$ grains near parallel to the tensile axis}

Fig. 2 shows HAADF images of ESFs in a $\langle 100\rangle$ grain along a [0 $\overline{1} 1]$ zone axis. The bottom left half of Fig. 2a depicts two Shockley partial dislocations marked by blue "L"s that are bounded by an ESF according to the identified stacking sequence going from the bottom upwards which is $A B C A|C| B C A B$. Interestingly, this ESF thickens by the glide of another Shockley partial dislocation in an adjacent (111) plane as observed in the top right half of Fig. 2a marked by a white "L". By following the sequence in the region to the right of the white "L" and going from the bottom upwards, the sequence $\mathrm{ABCA}|\mathrm{CB}| \boldsymbol{A B C A}$ can be observed which delineates a twin; such that $\mathbf{A}$ and $\boldsymbol{A}$ are the twin boundaries. This indicates that one of the twinning mechanisms operative in $\langle 100\rangle$ grains is based on the schematic proposed by Mahajan and Chin [14] (Refer to Fig. 17 in [5] for details). As stated earlier, it consists of the twin formation by the glide of co-planar Shockley partial dislocations on adjacent (111) planes following the reaction:

$a / 2[\overline{1} 10]+a / 2[01 \overline{1}] \rightarrow 3 a / 6[\overline{1} 2 \overline{1}]$

The reaction forms an ESF-ISF pair that subsequently thickens by the glide of a Shockley partial dislocation on adjacent $\{111\}$ planes. As more and more Shockley partial dislocations glide adjacent to each other, a macroscopic twin is formed. 


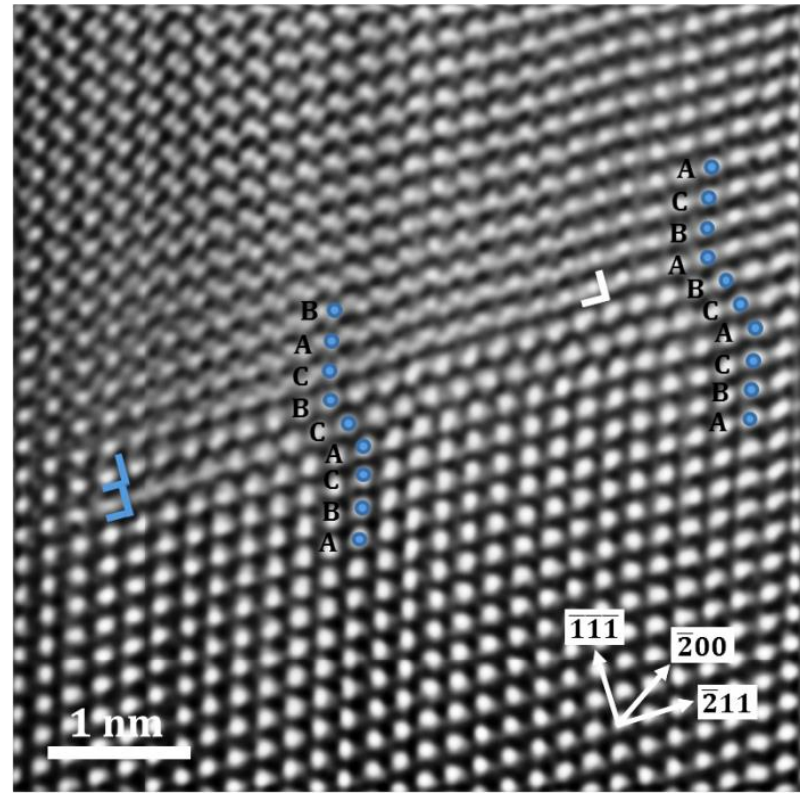

(a)

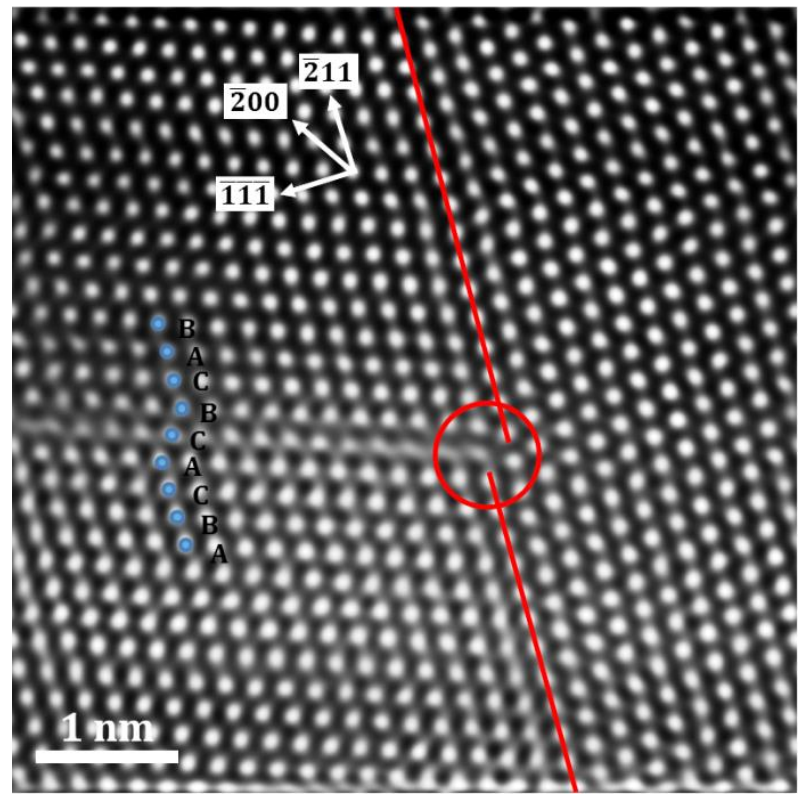

(b)

Figure 2: HAADF images of ESFs in a $\langle 100\rangle$ grain along the [0 $\overline{1} 1]$ zone axis. In (a) the two Shockley partial dislocations bounding the ESF are marked by blue "L"s. The Shockley partial dislocation bounding an ISF is marked by a white "L" and overlaps the ESF to form a twin. In (b) an ESF intersects a twin boundary marked by solid red lines. The twin boundary is displaced by one (111) plane due to the passage of a Shockley partial dislocation.

Further evidence of ESFs is provided in the HAADF image of a $\langle 100\rangle$ grain along the [110] zone axis (Fig. 2b). Here, an ESF is observed to interact with a twin boundary marked by solid red lines. By following the stacking sequence across the fault, going from the bottom to the top, the stacking sequence is $A B C A|C| B C A B ;$ which is consistent with an ESF. It should be noted that this sequence can also be formed by the subsequent glide of Shockley partial dislocations bounded by ISFs [9]. However, by looking at the interaction of the Shockley partial dislocation with the twin boundary, it is confirmed that the partial corresponds to an ESF as follows.

If a Shockley partial dislocation $a / 6[\overline{1} \overline{2} 1]$ glides on the (111) plane until it reaches the twin boundary, the partial can dissociate according to the following reaction:

$a / 6[\overline{1} \overline{2} 1] \rightarrow a / 6[\overline{1} \overline{1} 2]+a / 6[0 \overline{1} \overline{1}]$ 
As per the schematic in Zhu et al. [31] (Refer to Fig. 3 in that paper), the partial $a / 6[\overline{1} \overline{1} 2]$ would travel in the $[\overline{2} 11]$ direction and move the twin up by one close packed plane on the fault side of the twin boundary. Moreover, the stair-rod dislocation $a / 6[0 \overline{1} \overline{1}]$ can further dissociate into a partial $a / 2[11 \overline{2}]$ on the (111) plane and another partial $a / 6[\overline{1} \overline{2} 1]$ on the $(1 \overline{1} \overline{1})$ plane following the reaction:

$a / 6[0 \overline{1} \overline{1}] \rightarrow a / 6[11 \overline{2}]+a / 6[\overline{1} \overline{2} 1]$

The partial $a / 6[11 \overline{2}]$ translates the twin boundary up by one (111) plane on the other side of the fault. However, if we pay particular attention to the glide of the first $a / 6[\overline{1} \overline{1} 2]$, its glide was opposite to the direction reported in Ref. [31]. This is because the partial of an ESF has a Burgers vector $a / 6[12 \overline{1}]$ (which is the sum of the 2 partials shown in Fig. $2 \mathrm{~b}$ ) which dissociates as per the reaction:

$a / 6[12 \overline{1}] \rightarrow a / 6[011]+a / 6[11 \overline{2}]$

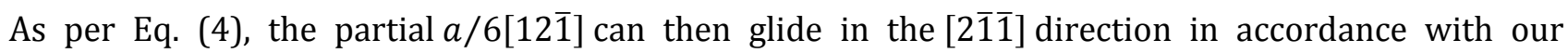
experimental results. The fact that the twin is displaced by one (111) plane is evidence that a Shockley partial dislocation bounded by an ESF interacted with a twin boundary.

\subsection{Twin growth by extrinsic stacking faults in $\langle 100\rangle$ grains near parallel to the tensile axis}

ESFs can not only serve as nuclei for twin formation but following Mahajan and Chin [14], can also contribute to the growth of twins. Fig. 3 shows two HAADF images of different twins marked by solid red lines growing by two $\{111\}$ planes due to the glide of Shockley partial dislocations bounded by ESFs

marked by blue "L"s. Fig. 3a depicts a twin grown from six to eight (111) planes. Although the size of the twin cannot be observed in Fig. 3b as the other twin boundary is not within the field of view, it is evident that it is being displaced (or grown) by two (111) planes. These observations are consistent with the glide of two Shockley partial dislocations bounded by an ESF on $\{111\}$ planes adjacent to twin boundaries. 


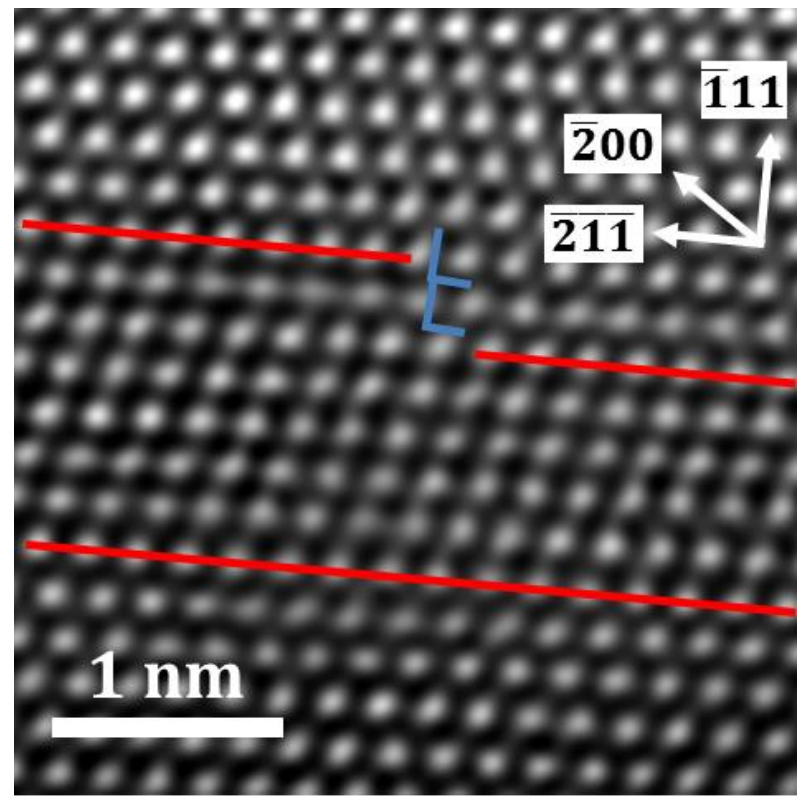

(a)

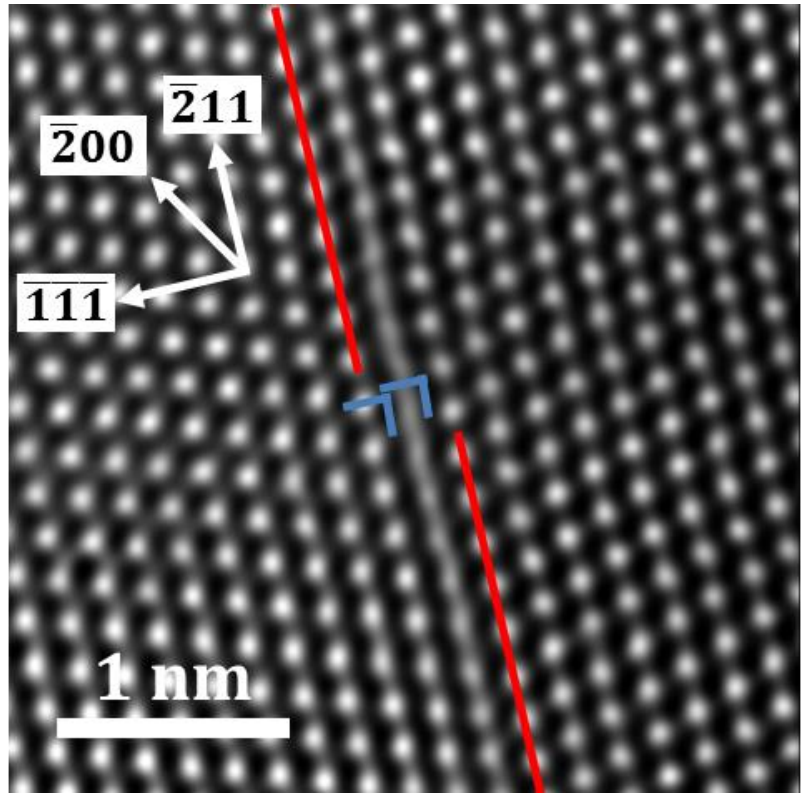

(b)

Figure 3: HAADF images of ESFs in a $\langle 100\rangle$ grain along the [0 $\overline{1} 1]$ zone axis contributing to twin growth. In (a) an ESF bounded by two Shockley partial dislocations marked by blue "L"s thickens a twin marked by solid red lines from six to eight ( $(111)$ planes. In (b) an ESF bounded by two Shockley partial dislocations marked by blue "L"s shift the twin boundary by exactly two (111) planes.

\subsection{Other twin nucleation mechanisms in $\langle 100\rangle$ grains near parallel to the tensile axis}

While ESFs were evidenced in $\langle 100\rangle$ grains as shown in Section 3.1, a host of other defects were also present in the low carbon TWIP steel uniaxially tensile loaded up to its ultimate tensile strength of $\sim 1080$ MPa and/or $48 \%$ true strain.

As a first representative example, the operation of the pole mechanism (a prismatic source) in $\langle 100\rangle$ grains was observed. Fig. 4a shows a $a / 3[111]$ Frank partial dislocation marked by a yellow "T" adjacent to an ISF. This is consistent with the pole-mechanism proposed by Venables [19]. As shown schematically in Fig. 15 of Ref. [5], the mechanism consists of a perfect dislocation that splits into Frank and Shockley partials; such that the Shockley partial then revolves around the Frank partial. 
In Fig. 4a, the presence of a Frank partial dislocation and the ISF (caused by the glide of the Shockley partial dislocation) is easily identifiable by following the stacking sequence above and below the Frank partial dislocation. In the top half of the figure, and going from left to right, the sequence is $A B C A \mid C A B C$ whereas in the bottom half of the figure the sequence is $\mathrm{ABC}|\mathrm{BA}| \boldsymbol{C A B C}{ }^{3}$. The stacking fault $\mathrm{A} \mid \boldsymbol{C}$ was most likely introduced by a Shockley partial dislocation whereas the extra plane B corresponds to a Frank partial dislocation.

As a second representative example, the operation of the synchronised activation of partial dislocations was observed. In Fig. 4b four-layer twin is shown with the sequence BACACBABC. As shown schematically in Fig. 4 of Ref. [32], the total Burgers vector of the Shockley partial dislocations forming the incoherent twin boundary of the four layered twin (marked by the red ellipse) is zero; thereby producing a net zero macrostrain.

Fig. 4b also shows sessile and glissile incoherent twin boundaries in $\langle 100\rangle$ grains. The glissile incoherent twin boundary (marked by a red ellipse in Fig. 4b) is mobile as it is formed only by Shockley partial dislocations. The sessile incoherent twin boundary is immobile as it features Shockley partial dislocations as well as sessile dislocations at the boundary; such as the Frank partial dislocation, with a Burger's vector $a / 3[\overline{1} 11]$, marked by a yellow “T” in Fig. 4b. The sessile Frank partial dislocation pins the incoherent twin boundary in position.

\footnotetext{
3 This sequence corresponds to a twin with one unit cell present on either side of planes $\mathbf{C}$ and $\boldsymbol{C}$ which are twin
} boundaries. 


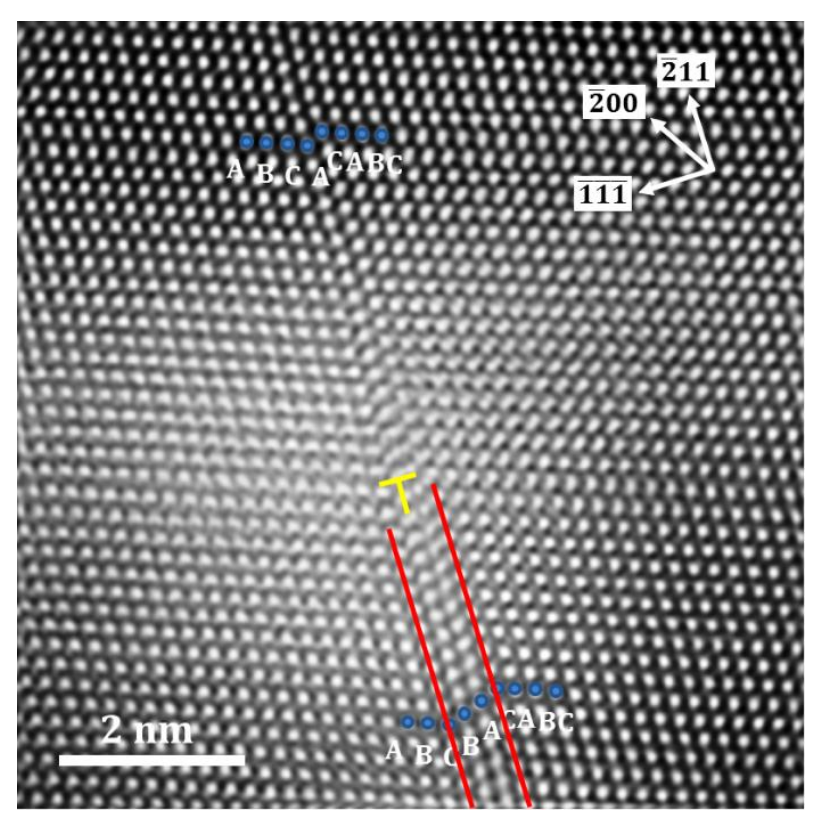

(a)

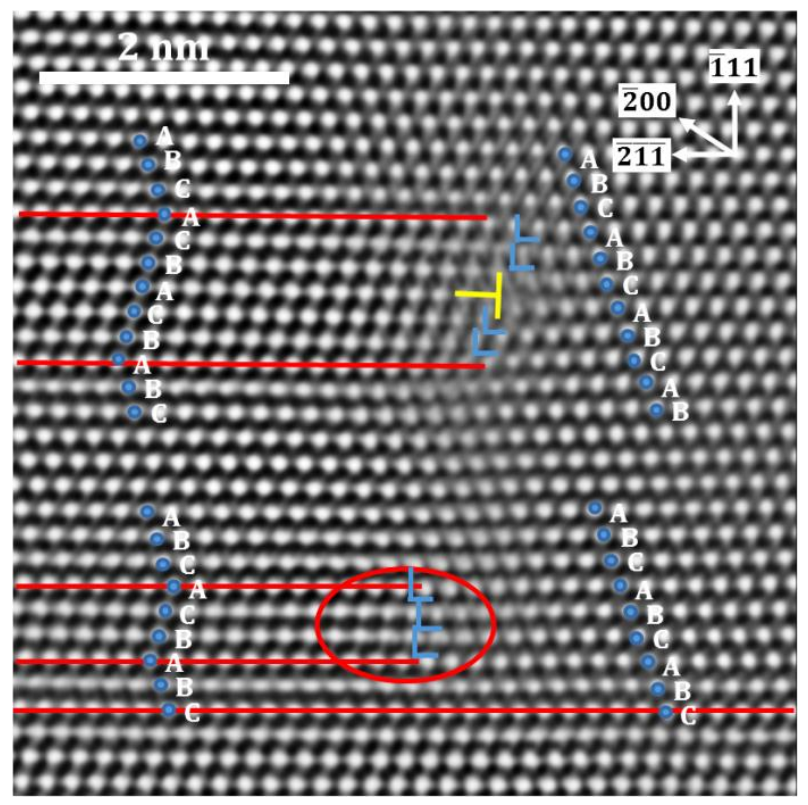

(b)

Figure 4: HAADF images of twins in a $\langle 100\rangle$ grain along the [0 $\overline{1} 1]$ zone axis. In (a) a Frank partial dislocation is marked by a yellow "T" and is shown next to an ISF forming a four-layered twin marked by solid red lines. In (b) a Frank partial dislocation, marked by a yellow "T", identifies the end of an immobile twin; Shockley partial dislocations are marked by blue "L"s. A mobile twin is identified by the interaction of only Shockley partial dislocations (marked by blue "L"s) at the end of the twin and is marked by a solid red ellipse.

\subsection{Twin nucleation in $\langle 111\rangle$ grains near parallel to the tensile axis}

Since $\langle 111\rangle$ grains are favourably oriented for twinning under uniaxial tension, well-developed twins are expected in these grains. Consequently, we investigated the twinning mechanisms in $\langle 111\rangle$ grains by examining crystal defects near their twin boundaries. A representative example of twin growth in $\langle 111\rangle$ grains is shown in Fig. 5a. Twin growth by one close packed plane on an adjacent $\{111\}$ plane occurred by the glide of a Shockley partial dislocation marked by a blue "L" bounding an ISF. 


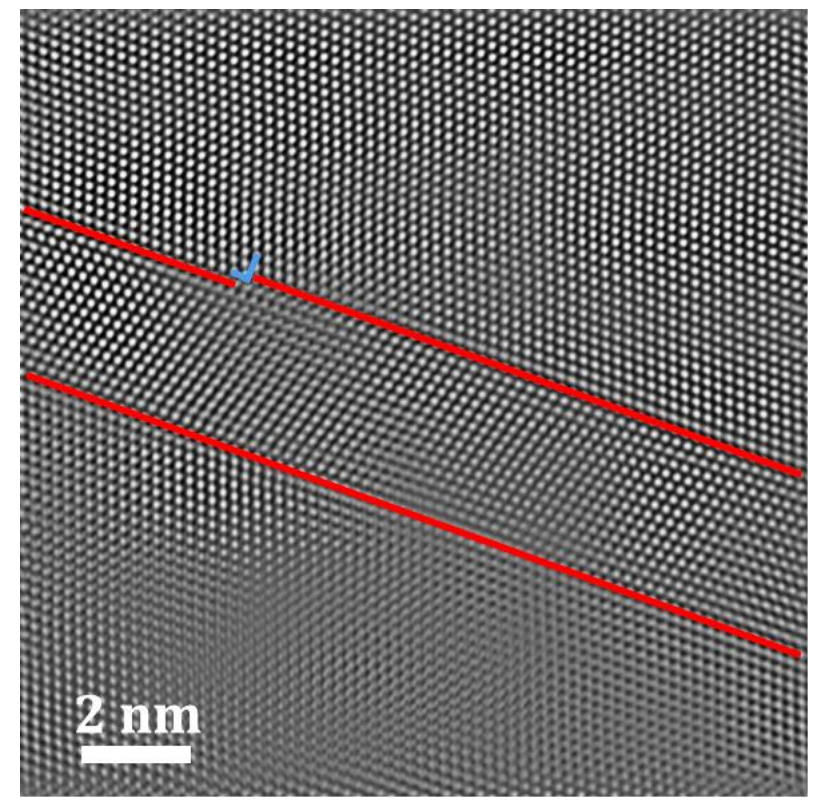

Figure 5: HAADF image of an ISF in a 〈111〉 grain along the [110] zone axis. An ISF bounded by a Shockley partial dislocation marked by a blue "L" is observed to shift a twin boundary marked by solid red lines by one $\{111\}$ plane.

\section{Discussion}

Stacking faults are usually assumed to be intrinsic in nature in fcc TWIP steels [9] due to the classical explanation by means of Schmid's law; wherein the values of the Schmid factor for twinning and the leading partial of ISFs are equivalent [22]. Consequently, previous Schmid factor calculations completely overlooked twin nucleation from extrinsic faults.

Tadmor et al. [33] define an ESF as a two-layered micro-twin or as a fault in the fcc matrix when a second Shockley partial dislocation is emitted on an adjacent (111) plane to a pre-existing ISF. As more consecutive Shockley partial dislocations are emitted in the following (111) planes, the micro-twin grows into a twin.

Following the above rationale, the density functional theory simulations assume that the barrier for the nucleation of ESFs, which is equivalent to a two-layered twin fault, is related to the difference between the energy barrier for twinning partial nucleation as compared to the energy barrier for the emission of a 
trailing partial dislocation [34-35]. When the difference is large, ESFs are thought of as relatively uncommon.

However, this rationale implies that twinning follows the sequence whereby a pre-existing ISF transforms to an ESF (or micro-twin), then to a three-layered twin and so on. Most importantly, the formation of an ESF is linked to the pre-existence of an ISF; which is different from the results shown in this study.

The formation of ESFs without pre-existing ISFs has been discussed in previous reports. For example, Yamakov et al. [36] showed the formation of ESFs without pre-existing ISFs and that they consisted of Shockley partial dislocations bounded by a double stacking fault. The formation mechanism is therefore fundamentally different to that described by Tadmor et al. [33].

In our view, the formation of an ESF without a pre-existing ISF is delineated when the Shockley partial dislocation at the end of the fault is unequivocally identified. With aberration corrected STEM and the strict application of Weertman's convention [30], we were able to identify the Shockley partial dislocations bounded by a double stacking fault; thereby confirming the formation of ESFs without preexisting ISFs.

The TEM imaging of $\langle 100\rangle$ grains in a low carbon TWIP steel uniaxially tensile loaded up to its ultimate tensile strength of $1080 \mathrm{MPa}$ and/or $48 \%$ true strain can be summarised as follows (Figs. 1-4): (i) ESFs serve as twin nuclei and also contribute to twin growth. (ii) Twinning via ISFs is also active and can be explained by the operation of the pole mechanism (a prismatic source) as suggested by Venables [19]. (iii) A twinning mechanism involving the synchronised activation of partial dislocations is also operative.

In TWIP steel the existence of both ISFs and ESFs was reported by Idrissi et al. [25]. However, the authors ascribed ESFs as precursors to $\varepsilon$-martensite phase transformation and not as nuclei for twinning. In a different report, Idrissi et al. [26] found ESFs after 2\% tensile strain and attributed the formation of twins to the stair-rod and cross-slip mechanisms [11]. Mahato et al. [37] reported the operation of the 
same two twinning mechanisms $[11,14]$ but with the predominant activation of primary twinning at $2 \%$ tensile strain through the glide of $a / 6\langle 121\rangle$ Shockley partial dislocations.

In the present study, while $\langle 111\rangle$ grains showed twin nucleation via ISFs, we did not observe a single ESF in such grains (Fig. 5). As a consequence, all of the above TEM evidence corroborates and validates our previous Schmid factor -based predictions on the feasibility of twinning nucleation via ISFs in $\langle 111\rangle$ grains and ESFs in $\langle 100\rangle$ grains [23].

With respect to the existence of ESFs in $\langle 100\rangle$ grains, their nucleation mechanisms deserve mention. Unlike Karaman et al. [21] who were able to delineate an extrinsic fault nucleation mechanism in [001] oriented single crystals of a Hadfield steel, four candidate mechanisms could be the causatives behind ESF formation in this study. The likely operation of any or all four nucleation mechanisms could be ascribed to our polycrystalline TWIP steel being uniaxially tensile loaded up to its ultimate tensile strength of $\sim 1080$ $\mathrm{MPa}$ and/or $48 \%$ true strain.

The first mechanism was proposed by Karaman et al. [21]. They postulated the formation of a Lomer-

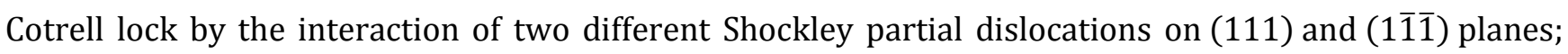
such that the lock acts as a high stress point. If the stress is high enough, the trailing partial will cross-slip onto the $(\overline{1} 1 \overline{1})$ plane. After further dissociating back into the (1 $\overline{1} \overline{1})$ plane, it forms an ESF. The ESF can be pinned down by interstitial atoms, forests of dislocations or kink formations [21]; which in turn causes the leading partial to be much more mobile than the trailing partial. Thereafter, the formation of a faulted loop occurs whereby the leading partial lags behind the trailing partial [21]. It is at this point that the leading

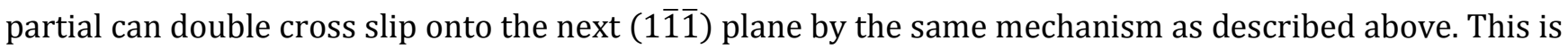
consistent with our observations of thin intersecting twins in $\langle 100\rangle$ grains (Fig. 6); which could have been the origin of the observed Shockley partial dislocations bounded by ESFs. 


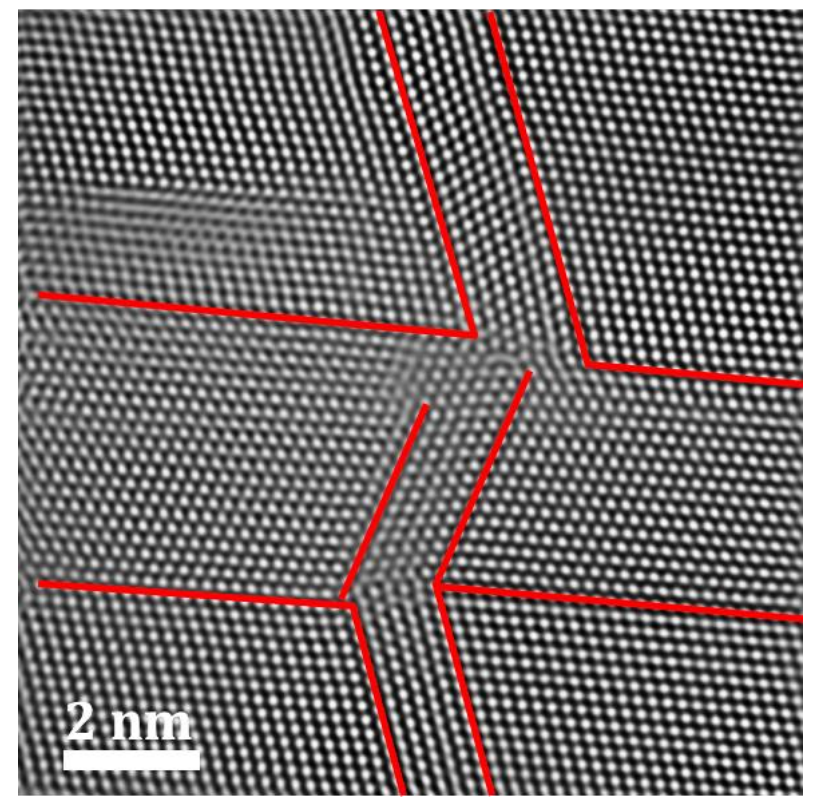

Figure 6: HAADF image of a $\langle 100\rangle$ grain along the [011] zone axis showing twin intersections. The solid red lines indicate twin boundaries.

The second mechanism was proposed by Mahajan and Chin [14] who suggested that thin twins nucleate from extrinsic-intrinsic fault pairs whose formation is said to occur as per Eq. (1). While this reaction is repulsive in nature, it is feasible in areas of high stress concentration. The twins could subsequently form by the overlapping of many ESFs on successive $\{111\}$ planes. While the fault pair should have been identifiable in atomic resolution HAADF images, we did not observe the formation of a single extrinsic-intrinsic fault pair. Since the TWIP steel was uniaxially tensile loaded up to its ultimate tensile strength of $\sim 1080 \mathrm{MPa}$ and/or $48 \%$ true strain, fault pairs could have recombined and reacted with other dislocations. Lastly, while dislocations bounded by ESFs were observed, the other end of the fault could not be analysed. Therefore there is still a possibility that they were present.

The third mechanism was observed in molecular dynamics simulations of nano-sized Al grains [36] and comprises the direct dissociation of a perfect dislocation directly to Shockley partial dislocations bounded by ESFs. However, this mechanism is deemed unlikely to operate due to a kinematic barrier to their formation [38]. 
Alternatively, the fourth mechanism described in the same molecular dynamics simulations [36] is more likely and involves Shockley partial dislocations bounded by ESFs forming at grain boundaries. The latter may be consistent with the study of Gutierrez-Urrutia et al. [4] who claim that twins originate at grain boundaries due to the increased stress caused by the twins in adjacent $\langle 111\rangle$ grains. However, they attribute this behaviour to a deviation from Schmid's law. On the other hand, if this fourth mechanism is operational, we suggest the possibility of ESFs nucleating at grain boundaries; a proposition that is consistent with our Schmid factor based calculations and the observation of ESFs contributing to twin growth.

\section{Conclusions}

Using aberration corrected STEM we comprehensively demonstrate that ESFs serve as twin nuclei and also contribute to twin growth in $\langle 100\rangle$ grains of a low carbon TWIP steel. Unlike previous studies, the present observations of ESFs in $\langle 100\rangle$ grains at high tensile strains indicates that twinning in such grains is not necessarily a deviation from Schmid's law. In addition, $\langle 100\rangle$ grains evidenced twinning through ISFs operating via the pole mechanism as well as twinning via the synchronised activation of partial dislocations. The ESF nucleation mechanisms could involve the formation of a Lomer-Cotrell locks, the formation of extrinsic-intrinsic fault pairs, extrinsically dissociated slip dislocations and/or nucleation from grain boundaries.

\section{Acknowledgements}

This work was financially supported by the Australian Research Council - Discovery Project grant scheme (DP130101883) and the Engineering Materials Research Strength at the University of Wollongong. The JEOL JEM-ARM200CF was purchased with financial assistance provided by the Australian Research Council - Linkage, Infrastructure, Equipment and Facilities grant (LE120100104). 


\section{References}

[1] P. Yang, Q. Xie, L. Meng, H. Ding, Z. Tang, Dependence of deformation twinning on grain orientation in a high manganese steel., Scripta Mater., 55 (2006) 629-631.

[2] R. Ueji, N. Tsuchida, D. Terada, N. Tsuji, Y. Tanaka, A. Takemura, K. Kunishige, Tensile properties and twinning behavior of high manganese austenitic steel with fine-grained structure, Scripta Mater., (2008) 963-966.

[3] D. Barbier, N. Gey, S. Allain, N. Bozzolo, M. Humbert, Analysis of the tensile behavior of a TWIP steel based on the texture and microstructure evolutions, Mater. Sci. Eng. A, 500 (2009) 196-206.

[4] I. Gutierrez-Urrutia, S. Zaefferer, D. Raabe, The effect of grain size and grain orientation on deformation twinning in a Fe-22 wt.\% Mn-0.6 wt.\% C TWIP steel, Mater. Sci. Eng. A, 527 (2010) 3552-3560.

[5] J.W. Christian, S. Mahajan, Deformation twinning, Prog. Mater Sci., 39 (1995) 1-157.

[6] A. Ookawa, On the mechanism of deformation twin in fcc crystal, J. Phys. Soc. Jpn., 12 (1957) 825-825.

[7] A.H. Cottrell, B.A. Bilby, LX. A mechanism for the growth of deformation twins in crystals, London, Edinb. and Dublin Phil. Mag. and J. Sci., 42 (1951) 573-581.

[8] J.A. Venables, Deformation twinning in face-centred cubic metals, Phil. Mag., 6 (1961) 379-396.

[9] J.P. Hirth, J. Lothe, Theory of dislocations, 2nd edition, John Wiley, New York, 1982.

[10] J.B. Cohen, J. Weertman, A dislocation model for twinning in f.c.c. metals, Acta Metall., 11 (1963) 996998.

[11] T. Mori, H. Fujita, Dislocation reactions during deformation twinning in $\mathrm{Cu}-11 \mathrm{at} . \% \mathrm{Al}$ single crystals, Acta Metall., 28 (1980) 771-776.

[12] S. Miura, J.-i. Takamura, N. Narita, Orientation dependence of the flow stress for twinning in silver crystals, Trans. Jpn. Inst. Met., 9 (1968) 555-561.

[13] M. Niewczas, G. Saada, Twinning nucleation in Cu-8 at. \% Al single crystals, Phil. Mag. A, 82 (2002) 167-191.

[14] S. Mahajan, G.Y. Chin, Formation of deformation twins in f.c.c. crystals, Acta Metall., 21 (1973) 13531363. 
[15] N. Naeita, J. Takamura, Deformation twinning in silver-and copper-alloy crystals, Phil. Mag., 29 (1974) 1001-1028.

[16] S. Mahajan, M.L. Green, D. Brasen, A model for the FCC $\rightarrow$ HCP transformation, its applications, and experimental evidence, Metall. Trans. A, 8 (1977) 283-293.

[17] N. Thompson, Dislocation nodes in face-centred cubic lattices, Proc. Phys. Soc., Sect. B, 66 (1953) 481.

[18] S. Mahajan, Critique of mechanisms of formation of deformation, annealing and growth twins: Facecentered cubic metals and alloys, Scripta Mater., 68 (2013) 95-99.

[19] J.A. Venables, The electron microscopy of deformation twinning, Journal of Physics and Chemistry of Solids, 25 (1964) 685-692.

[20] I. Karaman, H. Sehitoglu, A.J. Beaudoin, Y.I. Chumlyakov, H.J. Maier, C.N. Tomé, Modeling the deformation behavior of Hadfield steel single and polycrystals due to twinning and slip, Acta Mater., 48 (2000) 2031-2047.

[21] I. Karaman, H. Sehitoglu, Y.I. Chumlyakov, H.J. Maier, I.V. Kireeva, Extrinsic stacking faults and twinning in hadfield manganese steel single crystals, Scripta Mater., 44 (2001) 337-343.

[22] I. Karaman, H. Sehitoglu, K. Gall, Y.I. Chumlyakov, H.J. Maier, Deformation of single crystal Hadfield steel by twinning and slip, Acta Mater., 48 (2000) 1345-1359.

[23] A.A. Gazder, A.A. Saleh, E.V. Pereloma, On the feasibility of twinning nucleation via extrinsic faulting in twinning-induced plasticity steel, Scripta Mater., 68 (2013) 436-439.

[24] H. Idrissi, K. Renard, L. Ryelandt, D. Schryvers, P.J. Jacques, On the mechanism of twin formation in FMn-C TWIP steels, Acta Mater., 58 (2010) 2464-2476.

[25] H. Idrissi, L. Ryelandt, M. Veron, D. Schryvers, P.J. Jacques, Is there a relationship between the stacking fault character and the activated mode of plasticity of Fe-Mn-based austenitic steels?, Scripta Mater., 60 (2009) 941-944.

[26] H. Idrissi, K. Renard, D. Schryvers, P.J. Jacques, TEM investigation of the formation mechanism of deformation twins in Fe-Mn-Si-Al TWIP steels, Phil. Mag., 93 (2013) 4378-4391.

[27] H. Idrissi, K. Renard, D. Schryvers, P.J. Jacques, On the relationship between the twin internal structure and the work-hardening rate of TWIP steels, Scripta Mater., 63 (2010) 961-964. 
[28] C.R. Weinberger, W. Cai, Plasticity of metal nanowires, J. Mater. Chem., 22 (2012) 3277-3292.

[29] Y.T. Zhu, X.Z. Liao, X.L. Wu, Deformation twinning in nanocrystalline materials, Prog. Mater Sci., 57 (2012) 1-62.

[30] J.R. Weertman, Elementary dislocation theory, Oxford University Press, Oxford, 1964.

[31] Y.T. Zhu, J. Narayan, J.P. Hirth, S. Mahajan, X.L. Wu, X.Z. Liao, Formation of single and multiple deformation twins in nanocrystalline fcc metals, Acta Mater., 57 (2009) 3763-3770.

[32] B.Q. Li, B. Li, Y.B. Wang, M.L. Sui, E. Ma, Twinning mechanism via synchronized activation of partial dislocations in face-centered-cubic materials, Scripta Mater., 64 (2011) 852-855.

[33] E.B. Tadmor, S. Hai, A Peierls criterion for the onset of deformation twinning at a crack tip, J. Mech. Phys. Solids, 51 (2003) 765-793.

[34] H. Van Swygenhoven, P.M. Derlet, A.G. Froseth, Stacking fault energies and slip in nanoscrytalline metals, Nat. Mater., 3 (2004) 399-403.

[35] K.R. Limmer, First-principles investigations of iron-based alloys and their properties, Doctoral Dissertations, (2014) 2347. http://scholarsmine.mst.edu/doctoral_dissertations/2347

[36] V. Yamakov, D. Wolf, S.R. Phillpot, H. Gleiter, Deformation twinning in nanocrystalline Al by molecular-dynamics simulation, Acta Mater., 50 (2002) 5005-5020.

[37] B. Mahato, S.K. Shee, T. Sahu, S. Ghosh Chowdhury, P. Sahu, D.A. Porter, L.P. Karjalainen, An effective stacking fault energy viewpoint on the formation of extended defects and their contribution to strain hardening in a Fe-Mn-Si-Al twinning-induced plasticity steel, Acta Mater., 86 (2015) 69-79.

[38] J.P. Hirth, R.G. Hoagland, Extrinsically dissociated dislocations in simulated aluminium, Phil. Mag. A, 78 (1998) 529-532. 